\title{
INFRARED AND X-RAY VARIABILITY OF CYG X-3
}

\author{
C. G. WY NN - W I LLIAMS* \\ California Institute of Technology, Pasadena, Calif., U.S.A.
}

\begin{abstract}
The $2.2 \mu \mathrm{m}$ flux from the infrared counterpart of Cygnus X-3 has been found to vary in phase with the X-ray flux with a $4.8 \mathrm{~h}$ period. The infrared observations imply a radius $\sim 1 R_{\odot}$ for the X-ray object and do not favor models involving a compact object such as a neutron star or a black hole.
\end{abstract}

A $4.8 \mathrm{~h}$ periodicity has been detected in the $2.2 \mu \mathrm{m}$ flux density from the infrared counterpart of Cygnus X-3. Coordinated measurements (Becklin et al., 1973) by the Caltech infrared group on the 200-in. telescope and by the Mullard Space Sciences Laboratory's X-ray package on the Copernicus satellite on 1973 July 9 showed that the infrared and $X$-ray fluxes vary in phase over the $4.8 \mathrm{~h}$ period, although their light curves are not identical (Figure 1). The infrared periodicity has been observed on several occasions; the ratio of maximum to minimum flux density appears to stay constant, even though the mean infrared flux density was seen to vary between observing periods. A similar behaviour has been noted in the X-ray emission.

The simplest and most attractive explanation for the $4.8 \mathrm{~h}$ periodicity is that Cyg $\mathrm{X}-3$ is an eclipsing binary system. From the period it may be deduced that the separation of the stellar centres is $1.4\left(M / M_{\odot}\right)^{1 / 3} R_{\odot}$, where $M$ is the total mass of the system. Since the radius of the infrared source cannot be greater than this and since a lower limit on the distance to the source is known from radio observations (Braes et al., 1973), a lower limit to the $2.2 \mu \mathrm{m}$ brightness temperature may be obtained:

$$
T_{\mathrm{B}}>6 \times 10^{6}\left(\frac{M}{M_{\odot}}\right)^{-2 / 3} \mathrm{~K} .
$$

It therefore may be deduced that for any reasonable total mass of the Cyg X-3 binary system the object seen at $2.2 \mu \mathrm{m}$ has a surface temperature higher than that of the photosphere of any normal star. Furthermore, given the very high estimated value for the infrared surface brightness, and given the similarity of the X-ray and infrared eclipse curves, it seems extremely probable that both the X-ray and infrared fluxes originate from the same hot object. For any reasonable value for the total mass of the system, this object has a radius of the order of $1 R_{\odot}$. The observations described in this paper therefore definitely do not favour models for Cyg X-3 involving a compact object such as a white dwarf, a neutron star or a black hole.

Infrared data taken on 1972 July 11 are shown on two time scales in Figure 2. The data on the left show a sharp cut off at about 0715 attributable to the $4.8 \mathrm{~h}$ periodicity. Half an hour later the outburst shown on an extended time scale on the right of Figure 2 occurred. It may be seen that this outburst lasted only about five

* Now at Mullard Radio Astronomy Observatory, Cambridge, United Kingdom. 


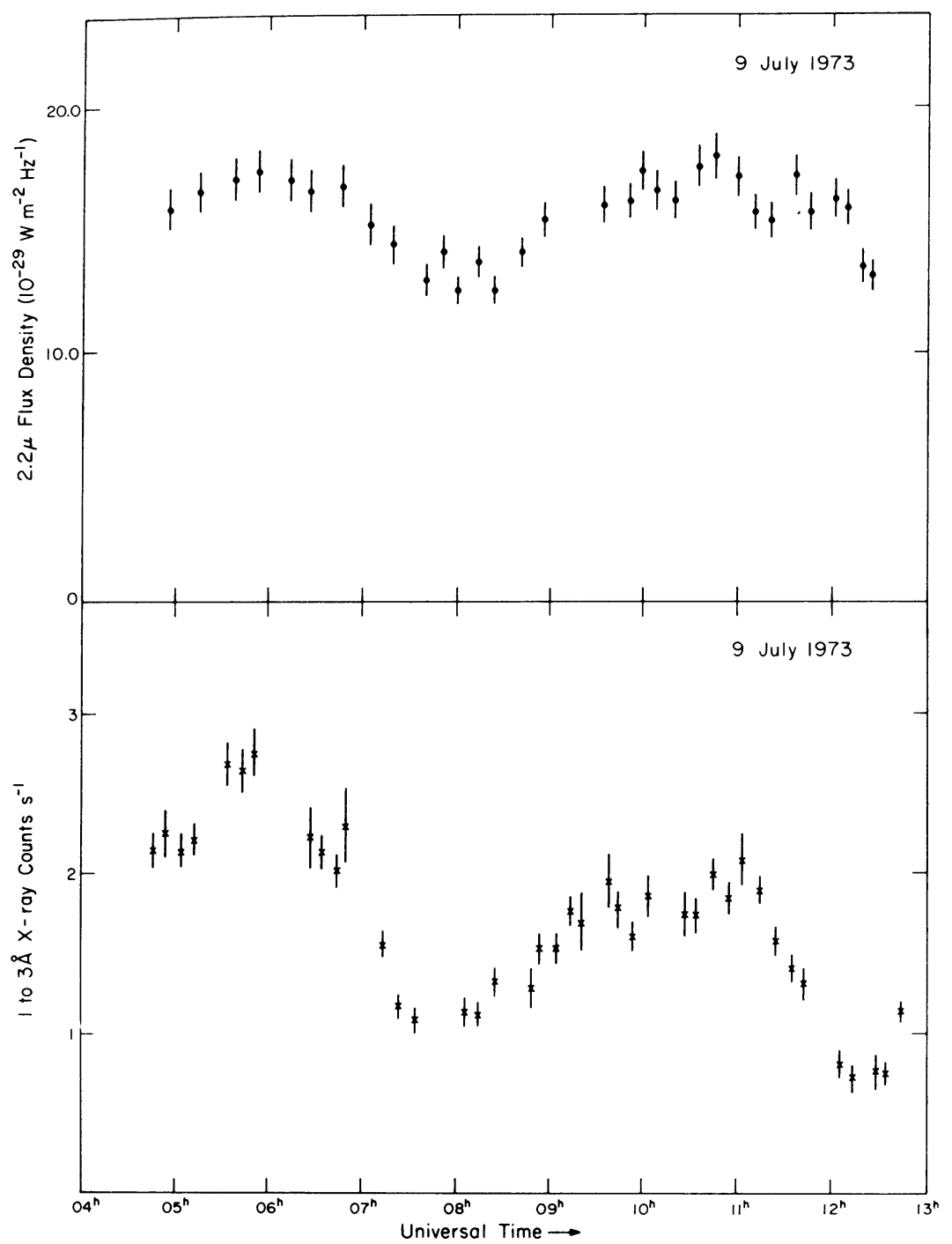

Fig. 1. The $4.8 \mathrm{~h}$ periodicity of Cyg X-3. The X-ray data have been averaged over the 6 min integration times of the infrared data. During the time period shown the infrared flux from a comparison star located within 5 " of Cyg X-3 appeared constant within $\pm 2 \%$.

minutes. Outbursts qualitatively similar to this one have been observed on other occasions; they do not appear to be correlated with the $4.8 \mathrm{~h}$ periodicity.

Although this erratic behaviour is highly reminiscent of the radio outbursts for which Cyg X-3 is notorious, it is not yet established how, if at all, the radio and infrared flares are related. Further observations to correlate X-ray, infrared and radio behaviour are in progress. 


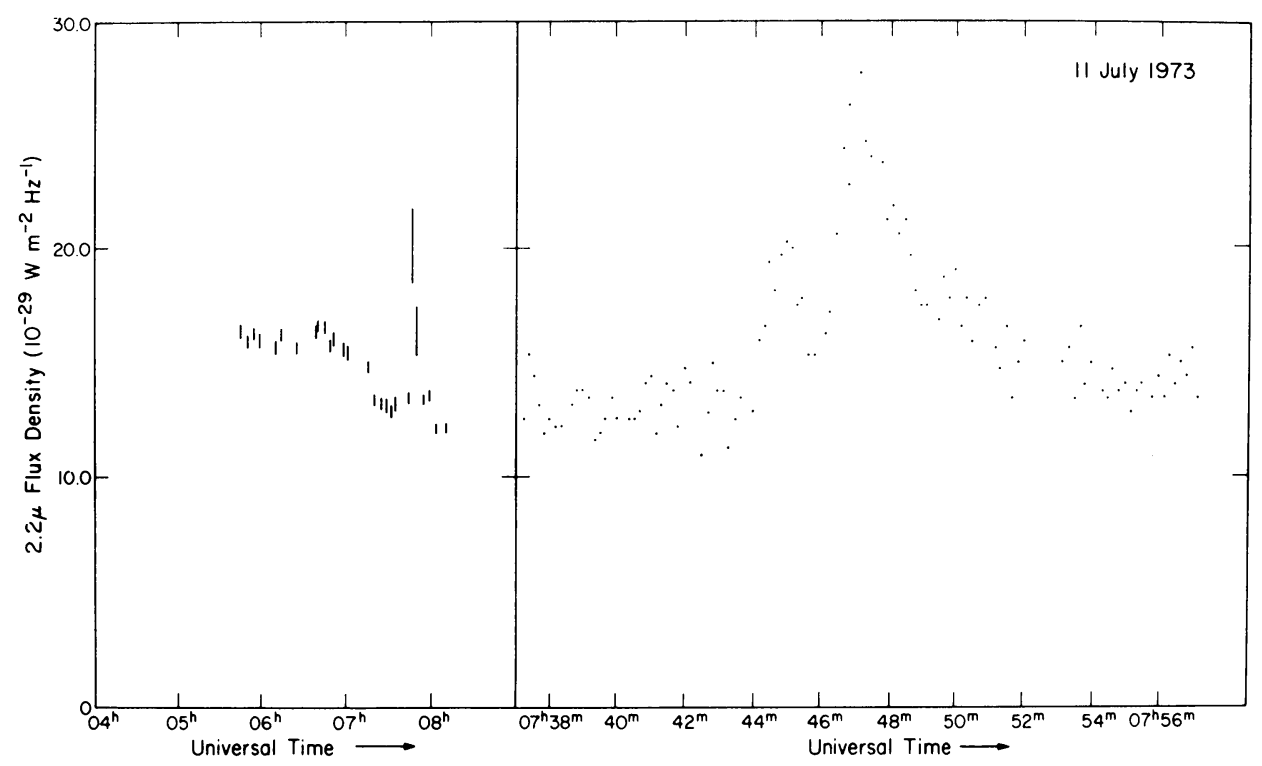

Fig. 2. The infrared flare of 1973 July 11. On the left hand side, the points represent 3 min integrations. A portion of the data at the left is shown broken up into $10 \mathrm{~s}$ samples. The statistical accuracy of each point is about $10 \%$.

\section{References}

Becklin, E. E., Neugebauer, G., Hawkins, F. J., Mason, K. O., Sanford, P. W., Matthews, K., and WynnWilliams, C. G. : 1973, Nature 245, 302.

Braes, L. L. E., Miley, G. K., Shane, W. W., Baars, J. W. M., and Goss, W. M.: 1973, Nature Phys. Sci. 242, 66.

C. G. Wynn-Williams

Mullard Radio Astronomy Observatory, Cavendish Laboratory, Free School Lane, Cambridge, CB2 3RQ, United Kingdom

\section{DISCUSSION}

Van Woerden: Would you say that from the fact that the flare does not come to a specific phase in the $4.8 \mathrm{~h}$ period, one would think of hot spots on the surface.

Wynn-Williams: No, one would not think of hot spots on the surface.

Fourikis: I would like to report that we observed radio emission from Henize 1044, yet another earlytype emission line object. Using the Parkes telescope at $3 \mathrm{~cm}$ the flux density is $242 \pm 34 \mathrm{Jy}$. This peculiar object is in many ways similar to HD 16362. This work was done in collaboration with C. R. Purton, P. A. Feldman, and A. E. Wright.

Westerhout: Hughes' picture of time variations as a function of frequency certainly reminds one of dynamic spectra of solar bursts, except for the time scale.

Hughes: This is true, but the three Cygnus X-3 bursts shown appear to be of different character, though all have the same rate of change of frequency with time. The different types of solar burst appear to have different values for $\mathrm{d} v / \mathrm{d} t$. 